\title{
HLA class II restricted helper and suppressor clones reactive with Mycobacterium leprae
}

\author{
RRP DE VRIES, T.H.M. OTTENHOFF, LI SHUGUANG \\ \& RA YOUNG \\ Department of Immunohaematology and Blood Bank, University Hos- \\ pital, Leiden, the Netherlands, and Whitehead Institute for Biomedical \\ Research, Cambridge, Mass., USA
}

\section{Introduction}

More than 10 years ago we decided to start a search for HLA-linked factors controlling the course of $M$. leprae infections (1). We choose an infectious disease because - in contrast to the diseases known to be associated with HLA at that time - there the etiological agent is known. We choose leprosy because of its remarkable spectrum of clinical symptoms paralelling the cell-mediated immune reactivity of the host to the bacillus (2). This choice appeared to be an extremely lucky one for two reasons. The first is that HLA molecules were shown to regulate antigen presentation to $\mathrm{T}$ cells (3), which apart from being important in leprosy appeared to be easy to clone in vitro (4). The second reason is that recently a remarkable progress has been made in the characterisation and synthesis of $M$. leprae antigens $(5,6)$. Thus we and others are now in a rather priviliged position to be able to study in detail the role of HLA products in the presentation of well defined medically relevant antigens. Such studies may contribute to the definition of both mechanisms and potential epitopes involved in protective immunity, immunopathology and suppression following an infection with M. leprae.

In this paper we will review our recent studies and present new data on restriction and antigen specificity of $M$. leprae reactive helper and suppressor clones.

\section{LEPRAE REACTIVE HELPER T CELL $\left(\mathrm{T}_{\mathrm{H}}\right)$ CLONES}

$M$. leprae reactive helper $\mathrm{T}\left(\mathrm{T}_{\mathrm{h}}\right)$ cells are probably responsible both for acquired protective immunity to the bacillus and delayed type hypersensitivity which may result in immunopathology $(2,7)$ : In order to obtain detailed information on the $M$. leprae epitopes recognized by $\mathrm{T}_{\mathrm{H}}$ cells and the HLA restriction determinants involved we decided to clone $M$. leprae reactive $\mathrm{T}_{\mathrm{H}}$ from the peripheral blood of leprosy patients.

One of the major technical obstacles in cloning $\mathrm{T}$ lymphocytes is the limited availability of autologous or HLA identical peripheral blood mononuclear cells, which are needed for restimulating established clones. This holds true especially for patients. Recently it was shown that Epstein-Barr virus transformed human B cell lines (EBV-BLCL) can process and present soluble antigens to $\mathrm{T}$ cells $(8)$. We have shown that this is also the case for $M$. leprae antigens (9). Such EBV-BLCL constitute an endless, continuous and homogeneous source of antigen presenting cells (APC's), which not only made T cell cloning much more feasible but also enabled us to study in detail the HLA molecules and epitopes that are used to present $M$. 
leprae antigens. The method we used to generate $M$. leprae reactive $\mathrm{T}_{\mathrm{H}}$ clones has been described previously (10). In brief, we isolated peripheral blood mononuclear cells (PBMNC's), generated EBV-BLCL and restimulated the PBMNC with Dhamendra lepromin. $T$ cell blasts were then cloned by limiting dilution on a feeder cell mixture consisting of irradiated autologous EBV-BLCL and PBMNC from random donors together with Dharmendra lepromin.

The $M$. leprae reactive clones thus obtained were of the $\mathrm{T} 3+\mathrm{T} 4+\mathrm{T} 8$ - phenotype and strongly HLA-DR positive. Upon addition of Dharmendra- or armadillo-derived lepromin and autologous APC's they strongly proliferated (measured as incorporation of radiolabeled thymidine) and produced- $\gamma$-interferon (IFN- $\gamma$ ). Based upon their membrane markers, IFN- $\gamma$ production, class II restricted proliferative responses to $M$. leprae antigens and the inability to suppress other $M$. leprae reactive $\mathrm{T}$ cells (vide infra) these $\mathrm{T}$ cell clones were defined as $\mathrm{T}_{\mathrm{H}}$ cells.

None of the $M$. leprae reactive $\mathrm{T}_{\mathrm{H}}$ clones reacted with unrelated antigens such as tetanus toxoid and candida. To assess whether these clones might recognize $M$. leprae specific determinants, 20 different mycobacterial preparations were presented to them (4). Four patterns of reactivity were observed: 1) $M$. leprae specific (roughly $25 \%$ of the clones), 2) crossreacting with one or two other mycobacteria, mainly $M$. vaccae and $M$. lepraemurium (another $25 \%$ ) 3) reactive with the majority of but not all mycobacteria tested (5-10\%), and 4) reactive with all mycobacterial preparations tested, sometimes with the exception of $M$. nonchromogenicum (nearly half the number of clones tested). Thus roughly $50 \%$ of the $\mathrm{T}_{\mathrm{H}}$ clones was absolutely or almost $M$. leprae specific.

In Table 1 we have summarized the results of $14 \mathrm{~T}_{\mathrm{H}}$ clones which were studied for reactivity against 3 of the $5 \mathrm{M}$. leprae proteins known to contain $M$. leprae specific epitopes seen by murine monoclonal antibodies (5).

Table 1. Helper $T$ cell clones from patients are reactive with the known $M$. leprae proteins

$\begin{array}{ll}\text { Reactive with } 65 \mathrm{~K} \text { and/or } 36 \mathrm{~K}: & \mathrm{n}=11 \\ \text { Reactive with only } 12 \mathrm{~K}: & \mathrm{n}=1 \\ \text { Not reactive with } 65 \mathrm{~K}, 36 \mathrm{~K}, \text { or } 12 \mathrm{~K}: & \mathrm{n}=2\end{array}$

It is clear that the majority of the clones recognize one or more of the proteins tested, and from results to be described below we infer that maybe all $M$. leprae reactive $T_{H}$ clones might turn out to be reactive with one or more of the 5 known $M$. leprae proteins. Of course this does not imply that these $T_{H}$ cells recognize the same epitopes as the mouse immunoglobulins. In fact we have evidence for the opposite: $\mathrm{T}_{\mathrm{H}}$ cell responses cannot be blocked by the relevant monoclonal antibodies.

One of the major breakthroughs research has been the cloning and expression of $M$. leprae DNA followed by screening with the above mentioned monoclonal antibodies recognizing $M$. leprae specific epitopes (6). Thus basically unlimited quantities of well-defined $M$. leprae proteins and peptides will become available in the near future. Some of our $\mathrm{T}_{\mathrm{H}}$ clones, obtained from patients were tested against $E$. coli lysates containing recombinant $M$. leprae proteins or at least peptides reactive with monoclonal antibodies against respectively $65 \mathrm{~K}$, 


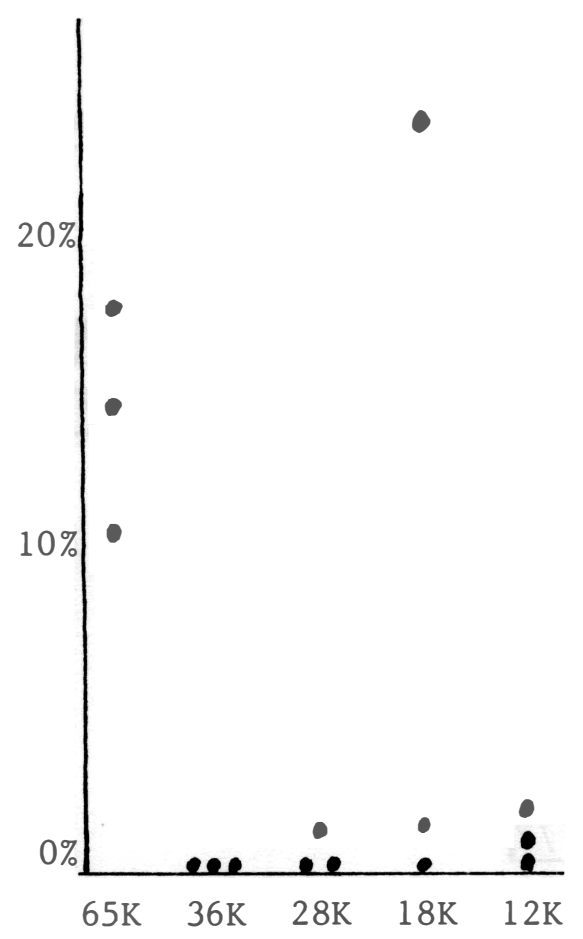

PATIENTS

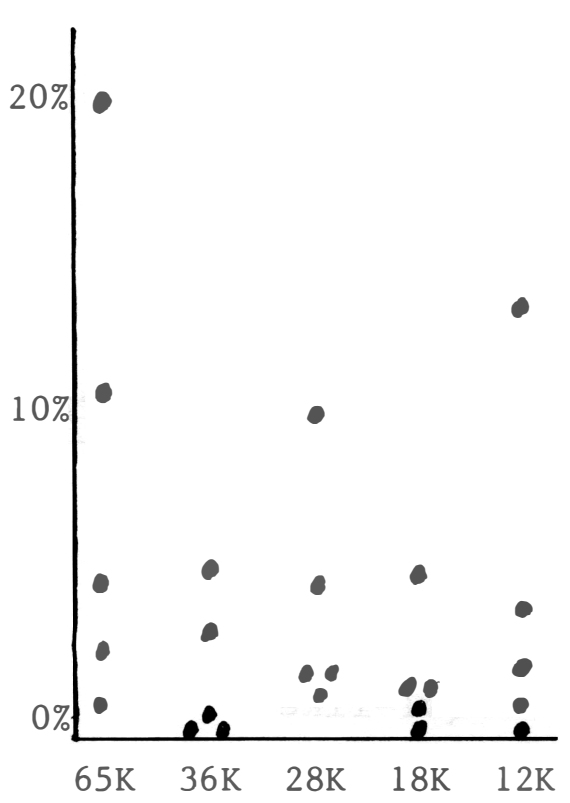

HEALTHY CONTACTS

Figure 1: $T$ cell reactivity with recombinant $M$. leprae proteins.

Expressed as percentage of $\Delta$ counts/min. to Dharmendra lepromin. $3 \mathrm{~T}_{\mathrm{H}}$ cell lines from tuberculoid patients and $5 \mathrm{~T}_{\mathrm{H}}$ cell lines from healthy contacts were tested with the $E$. coli lysates containing resp. $65 \mathrm{~K}, 36 \mathrm{~K}, 28 \mathrm{~K}, 18 \mathrm{~K}$ and $12 \mathrm{~K}$ proteins (ref. 6).

$36 \mathrm{~K}, 28 \mathrm{~K}, 18 \mathrm{~K}$ and $12 \mathrm{~K} \mathrm{M}$. leprae proteins. The first results were disappointing because few clones reacted, but on the other hand interesting because the positive reactions were only with the $65 \mathrm{~K}$ containing lysate. We thought this might be of potential interest because Mustafa et al. using the same preparations had shown that $\mathrm{T}_{\mathrm{H}}$ clones generated from healthy vaccinated subjects only reacted with the $18 \mathrm{~K}$ containing lysate (11). This observation suggested that $M$. leprae reactive $\mathrm{T}_{\mathrm{H}}$ cells from patients might recognize other epitopes than those from healthy contacts or successfully vaccinated subjects. In other words: this might provide a clue to the definition of epitopes providing protective immunity. In order to approach this question we decided to study bulk $T_{H}$ cell lines from several individuals rather than $T_{H}$ clones from one or at most a few individuals. The first results from $\mathrm{T}_{\mathrm{H}}$ cell lines obtained from 3 different (tuberculoid) leprosy patients and 5 healthy contacts are shown in Fig. 1. Although clearly more data would be neccessary, these data already suggest several interesting conclusions. In the first place they do not give further support to the idea the $18 \mathrm{~K}$ protein would contain epitopes of particular importance for protective immunity. Secondly, the $64 \mathrm{~K}$ recombinant protein containing lysate seems to be the best in vitro stimulator of (activated) $\mathrm{T}_{\mathrm{H}}$ 
MoAb

specificity

$\mathrm{DR}+\mathrm{DP}+\mathrm{DQ}$

DR

DRw52-like

DRw53-1ike

HLA-class I

DQ

DP

DQw1-like

DQw3-1ike
\% inhibition

$\underline{0} \quad 100$

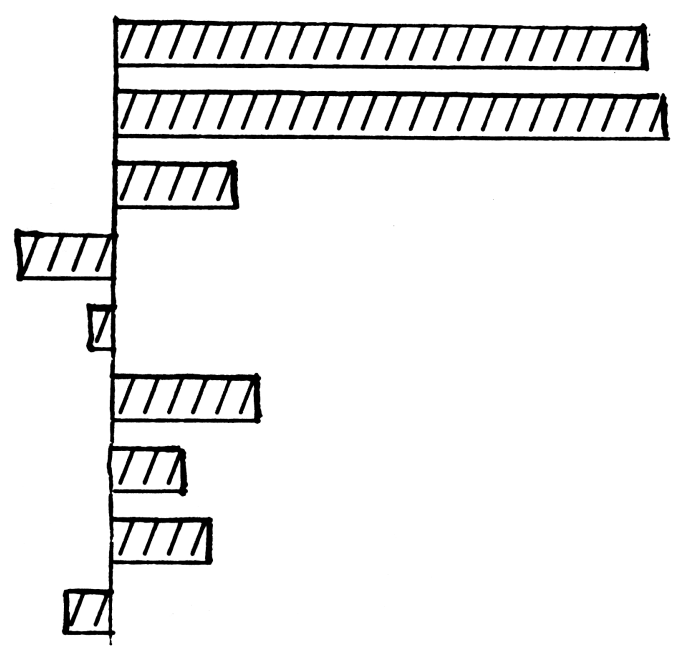

Figure 2: M. leprae antigens are presented to helper $T$ cells by DR molecules.

Inhibition of M. leprae induced response in the presence of a autologous APC. Results from a representative $T_{H}$ clone restricted by the DR4/Dw13 haplotype are shown. The results are expressed as percentage inhibiton of the $T_{H}$ response in the absence of monoclonal antibody (MoAb). Standard deviations did not exceed $10 \%$.

cells. Whether this also implies that the $65 \mathrm{~K}$ is the most immunogenic protein or contains the most important $\mathrm{T}_{\mathrm{H}}$ epitopes is too early to conclude given the lack of characterisation of at least some of the other recombinant proteins as well as other (possibly suppressive) components of the lysates (see other contributions in this issue). Finally, $T_{11}$ lines from patients tend to proliferate more consistently to the $65 \mathrm{~K}$ lysate than similar lines from healthy contacts. This might suggest that patients do recognize different determinants compared with healthy contacts.

HLA class II molecules are neccessary for and regulate the presentation of antigens to $T_{H}$ cells (12). The polymorphism of these HLA class II molecules may thus result in genetically controlled differences in $\mathrm{T}$ cell dependent immune responses. This might also be the case in leprosy: HLA class II linked genes are known to control the type of leprosy which develops upon infection (rather than the susceptibility to leprosy per se) as well as the in vivo cell mediated immune reactivity against $M$. leprae and related mycobacteria $(13,14)$. Since $T_{11}$ cells are of main importance in determining the cellular immune reactivity to $M$. leprae, the polymorphism of HLA class II gene products might thus be responsible for differences in presentation of $M$. leprae antigens to $\mathrm{T}_{\mathrm{H}}$ cells and thus control leprosy type. 
In order to study which HLA molecules and epitopes are important in the presentation of $M$. leprae antigens to $\mathrm{T}_{\mathrm{H}}$ cells, we have performed extensive panel and inhibition studies with fully HLA class II typed allogeneic antigen presenting cells and well defined HLA class II specific monoclonal antibodies (manuscript submitted for publication). The results of the blocking studies show in the first place that by far the majority (34 out of $36 \mathrm{~T}_{\mathrm{H}}$ clones) of restriction determinants (RD's) for $M$. leprae reactive $T_{H}$ clones are situated on DR (and not DP or DQ) molecules (fig. 2). These data obtained for clones from three leprosy patients were confirmed in studies with plyclonal $\mathrm{T}_{\mathrm{H}}$ lines obtained from 22 patients. Thus HLA-DR molecules seem to play a major role in the presentation of $M$. leprae antigens to $T_{H}$ cells. Since the expression of DR molecules usually is much stronger than that of DP and DQ molecules, this observation suggests that the capacity to present $M$. leprae (and probably other antigens) correlates with the quantitative expression of the different HLA class II molecules. This rule was confirmed, when we studied in more detail the restriction molecules on DR4 positive cells, which express two types of DR molecules called $\alpha \beta_{1}$ and $\alpha \beta_{3}$. The $\alpha \beta_{1}$ molecules carry the DR4 and Dw specificities and the $\alpha \beta_{3}$ molecules a supertypic determinant (DRw53) recognized by a monoclonal antibody. As shown in fig. 2 blocking studies mapped the RD's only on the $\alpha \beta_{1}$ (DRw53 negative) molecules, which are known to be highest in expression.

In order to study the fine specificity of the RD's for $M$. leprae antigens we performed large panel studies using selected allogeneic fully HLA class II typed APC's to present $M$. leprae antigens. A remarkable heterogeneity of the capacity to present $M$. leprae antigens was observed. For instance six different $M$. leprae reactive $\mathrm{T}_{\mathrm{H}}$ clones restricted via epitopes on the DR4-Dw 13 containing DR $\alpha \beta$, molecule showed 5 different patterns of responsiveness to the allogeneic APC's and M. leprae. Two patterns correlated with the DR4 and Dw 13 allospecificity, whereas the other three apparently correlated with different Dw 13 related epitopes. Because recently several DR $\beta_{1}$ alleles of the specificities studies by us have been sequenced, we were in a unique position to analyse the relation between structure and function of HLA class II molecules. The DR $\alpha \beta_{1}$ chains of Dw4, 13 and 14 positive individuals seem to differ for only 1-3 different nucleotides, which are likely to be situated on the outer face of the first domain of the DR $\beta_{1}$ molecule. Thus, like allo-reactive $\mathrm{T}_{\mathrm{H}}$ clones (15) our M. leprae reactive $T_{H}$ clones can distinguish between 1 till 3 amino acids difference between DR $\alpha \beta_{\text {I }}$ molecules. Moreover, they can apparently distinguish between the combination of as far as we know identical DR $\alpha \beta$, molecules and probably different $M$. leprae proteins or peptides. This leads to two important conclusions, at least one of which has practical implications. The first is that differences in processing of $M$. leprae (whatever that means) may contribute to the resulting $T_{H}$ repertoire. The second is that at least the DR4-Dw13 positive HLA DR $\beta_{\text {I }}$ molecule contains a large number of potential restriction determinants, and that most probably conformational changes of class II molecules contribute to the expression of them.

Finally, we have obtained preliminary evidence that allelic differences between DR molecules are correlated with differences in antigen specificity of DR-restricted $T_{H}$ clones: DR3-restricted $\mathrm{T}_{\mathrm{H}}$ clones from one tuberculoid leprosy patient recognized significantly more often than DR2-restricted $\mathrm{T}_{\mathrm{H}}$ clones $M$. leprae specific determinants. Note that DR3 is associated with tuberculoid leprosy in the population from which this patient originates. Of course such data will have to be confirmed in larger material and in different individuals. They may serve however as an example to show how the study of HLA class II Ir genes and their possible expression as restriction elements for antigen presentation to $T_{H}$ cells may contribute to define potential epitopes involved in protective immunity and immunopathology following infection with $M$. leprae. 


\section{LEPRAE REACTIVE SUPPRESSOR T CELL ( $\left.\mathrm{T}_{\mathrm{S}}\right)$ CLONES}

Whereas several important mysteries in cellular immunology, like the $\mathrm{T}$ cell receptor, have been solved during recent years, this does not seem to be the case for suppressor $\mathrm{T}$ cells. Therefore several immunologists assume that they do not exist in order to keep their schemes simple. We also like simple schemes, but we have recently cloned $\mathrm{T}$ cells from a borderline lepromatous leprosy patient which specifically suppress but do not kill autologous $T_{H}$ cells reactive with mycobacteria (16). This leaves us with two possibilities: either we have to show that we are dealing with an in vitro artefact or we have to show that such cells are important and - complicated or not - how they have to be fitted into at least the immunology of leprosy. At this stage we still favor the latter possibility.

Several investigators have implicated $\mathrm{T}_{\mathrm{S}}$ cells in the pathogenesis of the $M$. leprae specific $\mathrm{T}$ cell unresponsiveness observed in lepromatous leprosy patients. However, studies on this subject have been notoriously difficult to interpret for at least two reasons: in the first place the assays used to measure suppression were antigen non-specific or at least indirect and secondly heterogeneous cell population were used. Two recent reports $(16,18)$ may have the solution for both problems.

We have used the following approach: we observed that in contrast to $T$ cell lines from tuberculoid leprosy patients activated by $M$. leprae and propagated in IL-2, similar lines of (borderline) lepromatous leprosy consistantly failed to show a proliferative response against $M$. leprae antigens presented by autologous or allogeneic HLA class II matched APC. This lack of proliferation by thus cultured T cells was even observed when the PBMNC of such borderline lepromatous patients did proliferate to $M$. leprae. We selected one such a borderline patient arguing that this might enable us to study both $\mathrm{T}_{\mathrm{H}}$ and possible $\mathrm{T}_{\mathrm{S}}$ responses towards $M$. leprae. To our intense pleasure it appeared that the $M$. leprae non-responsive $\mathrm{T}$ cell line specifically suppressed the $M$. leprae response of autologous PBMNC's. Cloned T cells derived from this T cell line did not proliferate with $M$. leprae in the presence of APC but did suppress the response to $M$. leprae and other mycobacteria but not unrelated antigens. We could also generate $T_{H}$ clones from the same patient, some of which were also suppressed by the $\mathrm{T}_{\mathrm{S}}$ clones. All $\mathrm{T}_{\mathrm{S}}$ clones were $\mathrm{T} 3$ and most were positive for a $\mathrm{T}$ cell receptor framework determinant defined by monoclonal antibody WT31.

At present we do not know the mechanism of the specific suppression we observed. We do know that the $\mathrm{T}_{\mathrm{S}}$ clones are radiation sensitive. Trivial explanations like IL-2 consumption are ruled out by the antigen specificity, which is even observed when PBMNC are stimulated simultaneously with $M$. leprae and unrelated antigen. Also in such experiments only the $M$. leprae response is suppressed, indicating specificity at least in the effector phase. As shown in fig. 3, we have also excluded cytotoxicity of either APC or responding T cells.

An important question is whether the $\mathrm{T}_{\mathrm{S}}$ are activated by $M$. leprae antigens or by idiotypes of $\mathrm{T}_{\mathrm{H}}$ cells. If specialised epitopes (so-called suppressor epitopes) would be responsible for activating $T_{S}$ cells and not $T_{H}$ cells, suitable skin test and vaccine preparations might be developed which would contain only helper and no suppressor epitopes (18). On the other hand, if the suppression would be anti-idiotypic such a simple approach will probably not be useful (19). Thus far we have not obtained evidence for activation of $\mathrm{T}_{\mathrm{S}}$ clones by (idiotypes of) $T_{H}$ clones. On the other hand we did obtain evidence that the $T_{S}$ clones are activated by $M$. leprae antigens presented by APC in an HLA class II restricted fashion. This activation is blocked by anti-T3 antibodies and (in most cases) by the anti-T cell receptor antibody WT31.

So, if the $\mathrm{T}_{\mathrm{S}}$ clones described by us are relevant for the in vitro obserbed $M$. leprae specific suppression, we may now start to look for suppressor determinants on $M$. leprae antigens. The easiest situation would be that suppression is induced by non-protein determi- 


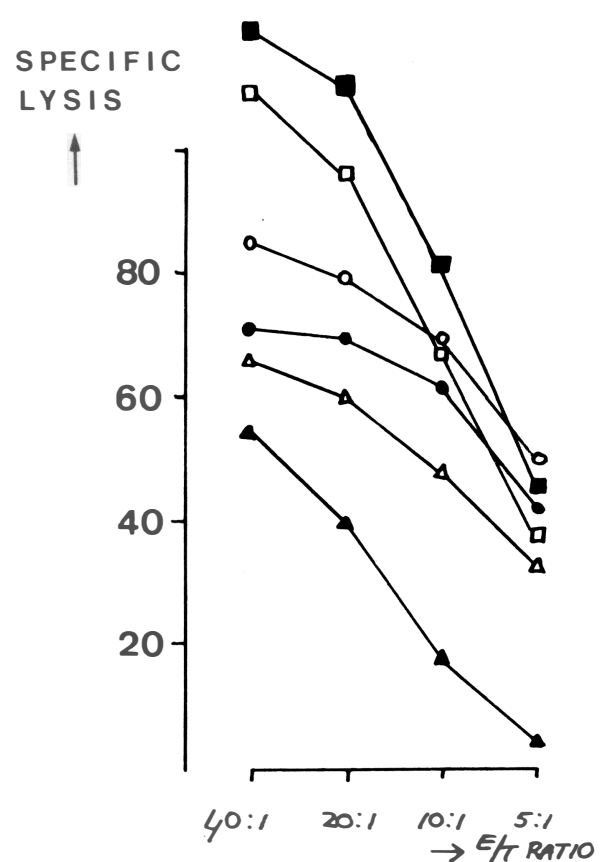

EFFECTOR
CTL LINE

$\propto A 2$
TARGETS :

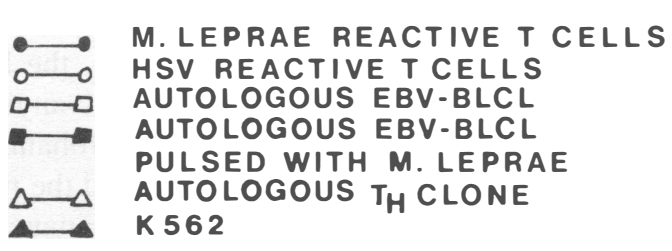

$\longrightarrow$ M. Leprae Reactive t CELls

HSV REACTIVE T CELLS

PULSED WITH M LEPRAE

AUTOLOGOUS $T_{H}$ CLONE

K 562

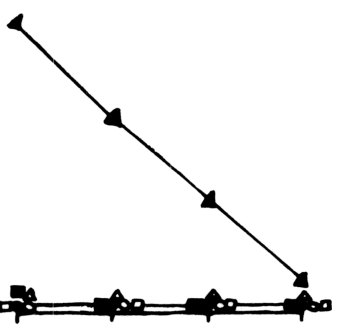

TS LINE
TS CLONES

$(N=5)$

Figure 3: $T_{S}$ clones are not cytotoxic.

$\mathrm{E} / \mathrm{T}$ ratio $=$ effector/target ratio.

nants, like the suppression described for $M$. leprae specific phenolic glycolipid (20). In contrast, we did obtain preliminary evidence that suppressor determinants are present on $M$. leprae proteins, e.g. the $36 \mathrm{~K}$ protein. Studies are now in progress which will answer the question whether helper and suppressor determinants on one $M$. leprae molecule may be dissociated or not.

As mentioned before, we obtained evidence that activation of $\mathrm{T}_{\mathrm{S}}$ clones is restricted by determinants on HLA class II molecules. Mixing experiments of allogeneic $T_{H}$ and $T_{S}$ clones yielded the same conclusion and moreover suggested that additional constraints apart from HLA class II restriction determinants are placed on suppression to occur. I hear Avrian Mitchison ask now: are your suppressor cells restricted by DQ? My tentative answer to this question is that at least some seem to be restricted by DR rather than DQ.

Of course most of the studies presented in this paper are far from final and a lot of work still needs to be done. We are sure that the clever study of $M$. leprae reactive $T_{H}$ and $T_{S}$ clones and lines from both patients and healthy contacts may offer important contributions to the search for mechanisms responsible for and epitopes involved in protective immunity, immunopathology and suppression during the course of an infection with $M$. leprae. We realise that for most of these studies the HLA restriction and the existence of Ir (and Is?) genes is only a nuisance. However, the study of HLA class II Ir and Is genes in this increasingly well defined system may - apart from being fun for a few immunogeneticists - also offer leads in the search aimed at better treatment and prevention of leprosy. 


\section{Acknowledgements}

We thank the leprosy patients and healthy contacts who donated the blood and in particular SC who even came several times for a lymphopheresis, as well as Drs. D. Leiker and R. Lai A Fat for their help in obtaining blood and clinical data. Antigens were kindly donated by Drs. R. Rees (IMMLEP M. leprae Bank), the late C. Shepard and R. Good (Atlanta), P. Klatser and A. Kolk (Amsterdam) and J. Ivanyi (London). John Haanen, Diënne Elferink, Jenny Cobessen, Saskia Neuteboom, Earl Johanns, Jos Pool and Joke Blom provided technical assistance. Tiny van Westerop prepared the manuscript. We thank Drs. B. Bloom and J. van Rood for their support of this project. Financial support was obtained from the Immunology of Leprosy (IMMLEP) component of the UNDP/World Bank/WHO Special Programme for Research and Training in Tropical Diseases, the Netherlands Leprosy Relief Association (NSL), the Foundation for Medical Research (MEDIGON grant 900-509-099), and the J.A. Cohen Institute for Radiopathology and Radiation Protection (IRS). Dr. Li Shuguang has a fellowship from the Chinese Ministery of Public Health.

\section{References}

1 De Vries RRP, Lai A Fat RFM, Nijenhuis LE, Van Rood JJ. HLA-linked genetic control of host response to Mycobacterium leprae. Lancet 2: 1328-1330, 1976.

2 Godal T, Myrvany B, Stanford JL, Samuel DR. Recent advances in the immunology of leprosy with special reference to new aspects in immunoprophylaxix. Bull. Inst. Pasteur 72: 247-310, 1976.

3 Doherty PC, Zinkernagel RM. A biological role for the major histocompatibility antigens. Lancet 1: 1406-1409, 1975.

4 Ottenhoff THM, Klatser PR, Ivanyi J, Elferink BG, De Wit MYL, De Vries RRP. Mycobacterium leprae specific protein antigens defined by cloned human helper T cells. Nature 319: 66-68, 1986.

5 Engers HD, Abe M, Bloom BR, Mehra V, Britton W, Buchanan TM, Khanolkar SK, Young DB, Closs O, Gillis T, Harboe M, Ivanyi J, Kolk AHJ, Shepard CC. Results of a World Health Organization - sponsored workshop on monoclonal antibodies to Mycobacterium leprae. Infect. Immun. 48: 603-605, 1985.

6 Young RA, Mehra V, Sweetser D, Buchanan TM, Clark-Curtiss J, Davis RW, Bloom $\mathrm{BR}$. Genes for the major protein antigens of the leprosy parasite Mycobacterium leprae. Nature 316: 450-452, 1985.

7 Bloom BR, Godal T. Selective primary health care: strategies for control of disease in the developing world. V. Leprosy. Rev. Inf. Dis. 5: 765-780, 1983.

8 Chen E, Umetsu D, Yunis E, Geha RS. EBV transformed B cell lines present to antigen specific $T$ cell clones determinants different from those recognized by antibody in an HLA-DR linked restricted fashion. Hum. Immunol. 8: 217-226, 1983.

9 Elferink BG, Ottenhoff THM, De Vries RRP. Epstein-Barr virus-transformed B cell lines present M. leprae antigens to T cells. Scand. J. Immunol. 22: 585-589, 1985.

10 Haanen JBAG, Ottenhoff THM, Voordouw A, Elferink BG, Klatser PR, Spits H, De Vries RRP. HLA class II restricted Mycobacterium leprae reactive $\mathrm{T}$ cell clones from leprosy patients established with a minimal requirement for autologous mononuclear cells. Scand. J. Immunol. 23: 101-108, 1986.

11 Mustafa S, Gill HK, Nerlund A, Britton WJ, Mehra V, Bloom BR, Young RA, Godal T. Human $\mathrm{T}$ cell clones recognize a major $M$. leprae protein antigen expressed in $E$. coli. Nature 319: 63-66, 1986.

12 Thorsby E. The role of HLA in T cell activation. Human Immunol. 9: 1-7, 1984. 
13 Van Eden W, De Vries RRP. HLA and leprosy: a re-evaluation. Lepr. Rev. 55: 89-104, 1984.

14 De Vries RRP, Van Eden W, Ottenhoff THM. HLA class II immune response genes and products in leprosy. Progr. Allergy 36: 95-113, 1985.

15 Cairns JS, Curtsinger JM, Dahl CA, Freeman S, Alter BJ, Bach FH. Sequence polymorphisms of HLA-DR $\beta_{1}$ alleles relating to T-cell-recognized determinants. Nature 317 : 166-168, 1985.

16 Ottenhoff THM, Elferink BG, Klatser PR, De Vries RRP. Cloned suppressor T cells from a lepromatous leprosy patient suppress Mycobacterium leprae reactive helper $\mathrm{T}$ cells. Nature 322: 462-464, 1986.

17 Bloom BR, Mehra V. Immunological unresponsiveness in leprosy. Immunol. Rev. 80: 5-28, 1984.

18 Oki A, Sercarz E. T cell tolerance studied at the level of antigenic determinants. I. Latent reactivity to lysosyme peptides that lack suppressogenic epitopes can be revealed in lysosyme-tolerant mice. J. Exp. Med. 161: 897-911, 1985.

19 Mitchison NA. Rational design of vaccines. Nature 308: 112-113, 1984.

20 Lymphocyte suppression in lepros induced by unique M.. leprae glycolipid. Nature 308 : 194-196, 1984. 